\title{
Temperature compensated silicon resonators for space applications
}

\author{
Mina Rais-Zadeh*, Vikram A. Thakar, Zhengzheng Wu, and Adam Peczalski \\ Department of Electrical Engineering and Computer Science, \\ University of Michigan, Ann Arbor, MI, USA 48109
}

\begin{abstract}
This paper presents piezoelectric transduction and frequency trimming of silicon-based resonators with a center frequency in the low megahertz regime. The temperature coefficient of frequency (TCF) of the resonators is reduced using both passive and active compensation schemes. Specifically, a novel technique utilizing oxide-refilled trenches is implemented to achieve efficient temperature compensation while maintaining compatibility with wet release processes. Using this method, we demonstrate high- $Q$ resonators having a first-order $\mathrm{TCF}$ as low as $3 \mathrm{ppm} /{ }^{\circ} \mathrm{C}$ and a turnover temperature of around $90{ }^{\circ} \mathrm{C}$, ideally suited for use in ovenized platforms. Using active tuning, the temperature sensitivity of the resonator is further compensated around the turnover temperature, demonstrating frequency instability of less than $400 \mathrm{ppb}$. Such devices are ideally suited as timing units in space applications where size, power consumption, and temperature stability are of critical importance.
\end{abstract}

Keywords: Clocks, flexural mode resonator, micromechanical resonators, TCF, temperature compensated, timing units, quality factor.

\section{INTRODUCTION}

Crystal resonators primarily using quartz are widely used for timing applications. However, current ultra-stable quartz resonators are large, making them unsuitable for employment in microsystems, such as those needed in small-size satellites. Despite recent efforts [1], [2], precision batch fabrication techniques are not capable of achieving nano-scale features in quartz and thus the clock frequency stability and system integration is severely limited. Silicon on the other hand is the material of choice for the majority of microelectronics applications and the concept of system-on-chip is primarily targeted towards using silicon as the common substrate. For this reason, the past decade has seen increasing effort to realize integrated microelectromechanical systems (MEMS) silicon resonators to replace quartz in timing applications [3]-[8]. MEMS resonators are most commonly actuated using capacitive or piezoelectric transduction mechanism. Quality factor $(Q)$ of capacitively transduced resonators is high due to the fact that they can be fabricated in a single, high-quality crystalline silicon layer. However, to achieve small motional impedance using capacitive transduction, very narrow gaps in the range of 1 to $100 \mathrm{~nm}$ are needed, reducing the RF power handling of the resonator and introducing large port parasitics [9]. On the other hand, the equivalent motional resistance of piezoelectrically transduced resonators is low [10]-[13]. For stable clocks, this is one of the most important characteristic as the phase noise of the clock and the frequency stability is directly related to the resonator driving power and the motional impedance. For this reason in this work, we target the use of piezoelectric transduction using aluminum nitride (AlN) and demonstrate AlN-on-silicon resonators for chip-scale timing units.

Although research into piezoelectrically actuated devices has been successful to the level that film bulk acoustic resonators (FBARs) using AlN are being incorporated in commercial products [14], several shortcomings still exist for micro and nanomechanical acoustic resonators for space applications: most important of which is the relatively large ( $60 \mathrm{ppm} /{ }^{\circ} \mathrm{C}$ ) temperature coefficient of elasticity (TCE) of silicon and AlN. It is essential to compensate for this large negative TCE (and the resulting TCF) within the resonator design itself in order to meet the stringent timing stability specifications for space applications. Degenerate doping [15] and use of materials with opposite TCF values [16] have both been explored as mechanisms to reduce the TCF value of such resonators. For example, using a thermally grown oxide coating, which has a positive TCF, a silicon resonator has been demonstrated with frequency variation of less than $200 \mathrm{ppm}$ over a wide temperature range [17]. A passive TCF compensation strategy utilizing oxide pillars uniformly distributed through the resonator was demonstrated in [18]. In order to make the fabrication compatible with wet release processes, we have recently implemented oxide-refilled trenches within the silicon resonator body to efficiently reduce

*minar@umich.edu; phone 1(734)764-4249; fax 1(734)763-9324; www.eecs.umich.edu/ minar 
the TCF [19]. We demonstrated that the position of the oxide-refilled trenches in the resonator body is critical towards maximizing temperature compensation with minimum material deposition and minimum $Q$ degradation. In this work, we show that by designing the location and geometry of the TCF compensation trenches, the first-order TCF of the resonators can not only be nullified but the temperature of zero TCF (i.e. the turnover temperature) can be tuned to the required operating temperature.

Using this TCF compensation technique, we demonstrated resonators with short term frequency drift in the order of 100 ppb [19]. In order to understand the cause of this frequency drift, we measure the stability of these resonators at their turnover temperature and at room temperature. Results show that the stability is significantly better when the device is operated at its turnover temperature which indicates that temperature fluctuations are predominantly responsible for the measured frequency instability. Thus improved clock performance can be achieved if the turnover temperature is designed to be close to the oven temperature. Such high-performance resonators can find numerous applications in different sectors ranging from space and military to consumer products.

\section{DESIGN AND FABRICATION}

\subsection{Device Principle}

Target specifications of the resonator are listed in Table 1. As the frequency stability of the resonator is related to its frequency and quality factor, a high-frequency (> $1 \mathrm{MHz})$, high- $Q$ design is targeted. As will be shown later, the resonator is more stable around its turnover temperature point. Inside an ovenized platform, it is thus desirable to set the turnover temperature to be around $70{ }^{\circ} \mathrm{C}$ to $90{ }^{\circ} \mathrm{C}$ (which is

Table 1. Target specification of the resonator.

\begin{tabular}{l|l}
\hline Parameter & Value \\
\hline Frequency & $>1 \mathrm{MHz}$ \\
\hline Resonator $\boldsymbol{Q}$ & $>10,000$ \\
\hline Turnover temperature & $70^{\circ} \mathrm{C}$ to $90^{\circ} \mathrm{C}$ \\
\hline Temperature stability & $<0.1 \mathrm{ppm}$ (oven controlled) \\
\hline
\end{tabular}

the usual temperature range of the oven). If the device is not temperature controlled and for consumer applications, of course a turnover temperature near room temperature is more desirable. The device structure used to meet these target specifications is shown schematically in Fig. 1(a). It is composed of two resonating flanges operated in their in-plane flexural mode, and connected together with a central rod. Two small tethers connect the center of the rod to the anchors. The structure of this device resembles the letter "I" and hence is called here after as an IBAR. Capacitively actuated IBARs with $Q$ s exceeding 100,000 have been reported previously [20], [21]. In a recent work [19], we demonstrated piezoelectric actuation of an IBAR in order to improve the motional impedance and obviate the fabrication complexity required to make narrow electrostatic actuation gaps. In order to resonate the IBAR in an in-plane flexural mode, the RF signal is applied to the input electrodes across both the flanges (Fig. 1(a)). The mode is actuated through the piezoelectric coefficient $d_{31}$, which converts the applied vertical electric field into a lateral stress gradient. The resonant frequency of this resonator can be approximated as

$$
f \cong 0.646 \frac{w}{L^{2}} \sqrt{\frac{E}{\rho}},
$$

where $E$ is the effective Young's modulus and $\rho$ is the effective density of the resonating stack. $w$ and $L$ are the width and the length of the resonating flange. In [19], we demonstrated that the TCF of AlN-on-silicon resonators can be reduced using oxide-refilled trenches (shown as green boxes in Fig. 1(a)) and the position of these oxide trenches has a direct

(a)

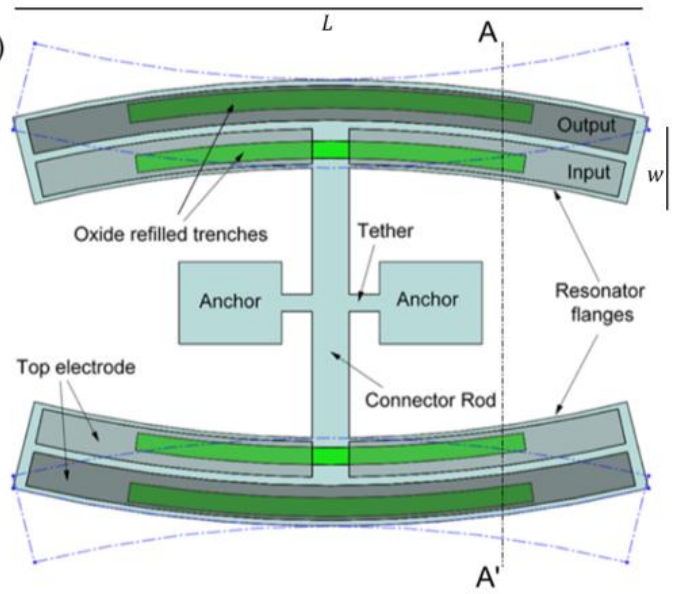

(b)

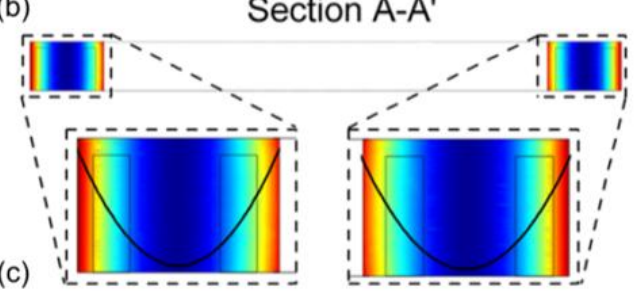

Figure 1. A cartoon showing (a) the mode shape and the structure of the resonator, (b) simulated strain energy density along section $\mathrm{A}-\mathrm{A}^{\prime}$, and (c) A close-up view of the strain energy density variation across the resonator flange cross-section. The overlaid parabolic curve (in black) represents the variation of the strain. 
impact on the final TCF value. Figures 1(b) and (c) plot the strain energy density across the section A-A'. From these simulation results, we can find that the regions of high stress are near the device edges in case of flexural modes. By placing oxide-refilled trenches close to the resonator edge, where strain energy is high, efficient temperature compensation can be achieved [19]. In this work we explore the tunability of the turnover temperature through oxide-refilled trench size modifications. Figure 2 plots the variation of turnover temperature with the change in the trench geometry assuming material parameters listed in Table 2 . Note that changing the width of the trenches will require changes in the oxidation time, as discussed in the next section, while changes in the trench length allows maintaining the same process parameters. As shown in Fig. 2, by varying the dimensions of the oxide-refilled trenches, the turnover temperature can be designed to be anywhere between $-40{ }^{\circ} \mathrm{C}$ to $80{ }^{\circ} \mathrm{C}$. Note that these dimensions can be comfortably defined using optical lithography.
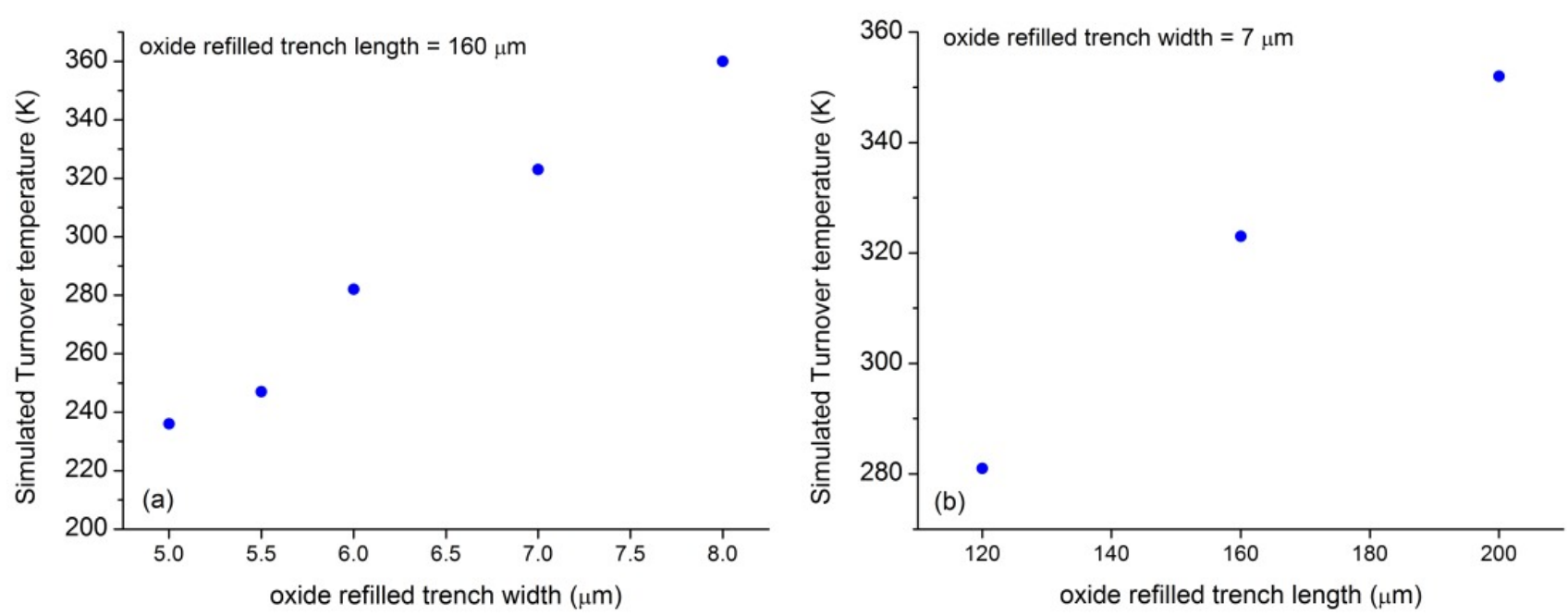

Figure 2. Simulated resonator turnover temperature of TCF as a function of the (a) oxide refilled trench width and (b) oxide refilled trench length. In all cases the trenches are offset from the edge of the resonator by $2 \mu \mathrm{m}$.

\subsection{Fabrication Process}

The fabrication process flow has been previously described in detail [19]. To summarize, the process starts with defining the temperature compensation trenches in a starting SOI wafer using deep reactive ion etching (DRIE). These trenches are subsequently refilled by growing thermal silicon dioxide at $1200{ }^{\circ} \mathrm{C}$. By etching a number of trenches next to each other with a specific spacing between them, wider oxide islands can be formed [19]. Post oxidation, the wafer surface is polished and the surface oxide is removed using chemical mechanical polishing (CMP). After CMP, the piezoelectric stack consisting of $100 \mathrm{~nm}$ molybdenum, $0.5 \mu \mathrm{m} \mathrm{AlN}$, and $100 \mathrm{~nm}$ gold is deposited and patterned. The contours of the resonator and tuning electrodes are defined by etching the AIN and the silicon device layer using DRIE. Finally, the device is released by selective backside removal of silicon and buried oxide layers. Figure 3 shows scanning electron micrograph (SEM) images of a $20 \mu \mathrm{m}$ thick piezoelectrically actuated silicon device.

\section{RESULTS AND DISCUSSION}

\subsection{Passive Compensation Results}

Figure 4 plots the measured peak frequency shift as a function of temperature for an uncompensated resonator and two passive compensation configurations. As shown, the TCF of the uncompensated resonator (with no oxide-refilled trenches) is close to the intrinsic TCF of high-resistivity silicon (i.e. $\sim-30 \mathrm{ppm} /{ }^{\circ} \mathrm{C}$ ). The structure of the compensated resonators is similar to the one shown in Fig. 1. The resonator with $5.5 \mu \mathrm{m}$ wide and $160 \mu \mathrm{m}$ long oxide-refilled trenches has a turnover temperature of $-20{ }^{\circ} \mathrm{C}$ (red triangle markers). Increasing the width of the oxide trench to $8 \mu \mathrm{m}$ while keeping the same trench length shifted the turnover temperature to $+90^{\circ} \mathrm{C}$ (blue circle markers). The results agree well 
with the simulation data provided in Fig. 2. Thus, by making modifications to the trench geometry and position, the turnover temperature can be tuned across a large temperature range.
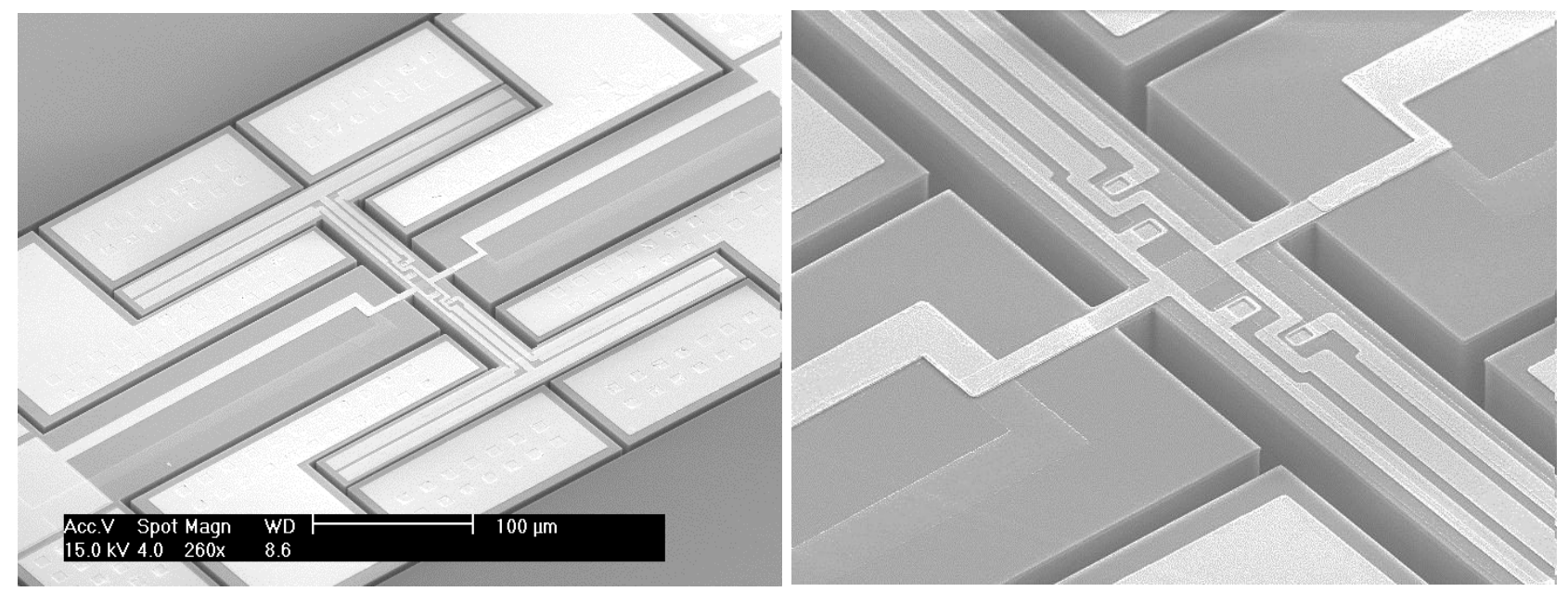

Figure 3. SEM images of a fabricated resonator. The vias through the AlN layer and the underpasses can be seen in the right image. The presence of the bottom electrode and the dielectric property of AlN allows us to easily create signal crossover required for the effective piezoelectric actuation of the in-plane flexural mode.

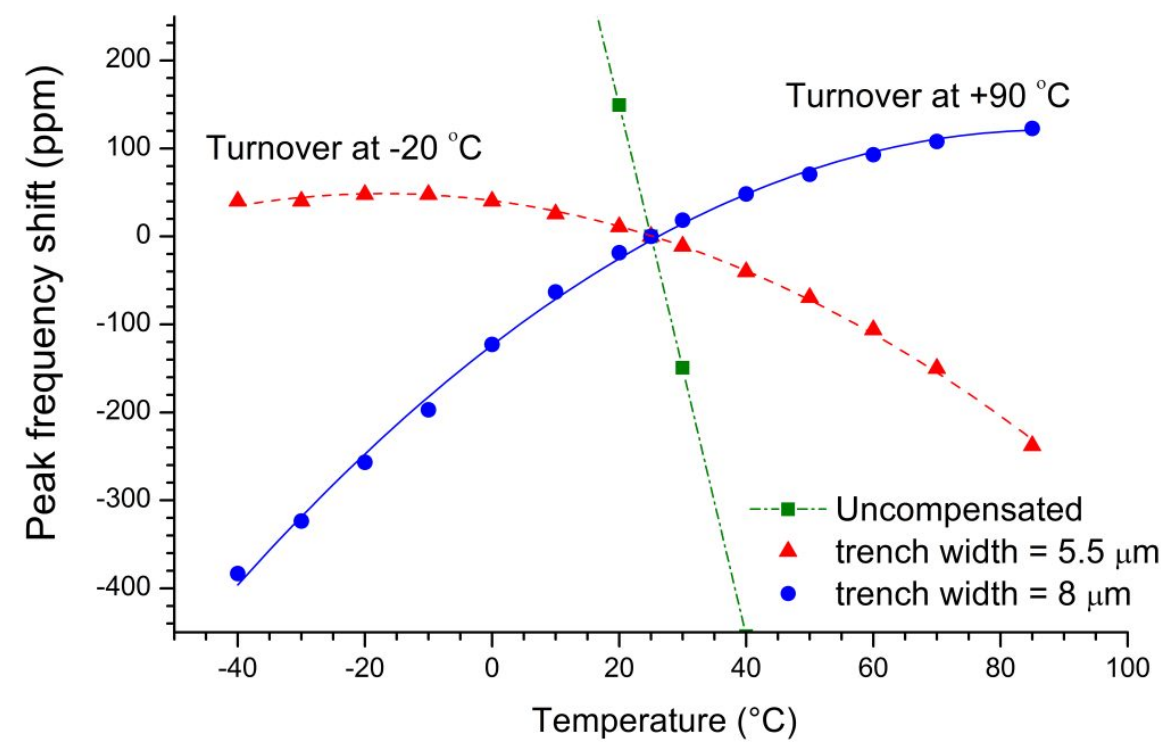

Figure 4. Measured peak frequency change for three AlN-on-silicon resonators with different oxide-compensation configuration. The $\mathrm{TCF}$ of the un-compensated resonator is $-30 \mathrm{ppm} /{ }^{\circ} \mathrm{C}$ (green square marker). The second resonator with $5.5 \mu \mathrm{m}$ wide and $160 \mu \mathrm{m}$ long oxide-refilled trenches has a turnover temperature of $-20{ }^{\circ} \mathrm{C}$ (red triangle markers). Increasing the width of the oxide trench to $8 \mu \mathrm{m}$ while keeping the same trench length shifted the turnover temperature to $+90{ }^{\circ} \mathrm{C}$ (blue circle markers). The lines in case of the two compensated resonators represent the second order polynomial fits to the measured data. Effective TCF of the second resonator at room temperature is $-3 \mathrm{ppm} /{ }^{\circ} \mathrm{C}$.

\subsection{Active Temperature Compensation Results}

Using passive compensation, the temperature induced frequency instability of the resonator can be reduced from $\sim 3600$ ppm to within $500 \mathrm{ppm}$ across a wide temperature range of $120{ }^{\circ} \mathrm{C}$ (Fig. 4). However, for resonator applications in timing references, this frequency variation is still too high. Operating the resonator in a temperature controlled platform is a commonly implemented strategy to mitigate this problem. In such a case, the clock stability is a function of the temperature stability of the oven. Considering that the oven control only has heating, implementing an optimum 
temperature control with a stable and quick settling time is a challenge. As a result, the oven temperature can show nonzero temperature variation for prolonged time intervals. In this time frame, active compensation can be used to stabilize the clock frequency and reduce the frequency instability associated with the oven temperature fluctuations.

Electrostatic tuning is commonly implemented to achieve this frequency trimming function and has been demonstrated previously [17], [19]. However, the use of electrostatic tuning requires the application of a DC bias during nominal operation which leads to increased power consumption and more importantly makes the clock frequency susceptible to bias voltage fluctuations. In this work we explore the piezoelectric tuning effect to actively compensate for fluctuations around the ovenization temperature. Since piezoelectric tuning is bidirectional, i.e. the frequency can either be increased or decreased depending on its polarity, nominal operation does not require any DC bias. Figure 5 plots the measured piezoelectric tuning for an IBAR. In order to measure the piezoelectric tuning effect, a DC bias is superimposed on top of the input and output RF signals. The applied electric field is only between the top electrode (applied DC bias) and bottom electrode (DC ground) layers. Since the thickness of the AlN layer is $2.44 \%$ of the total thickness, the tuning coefficient is quite small and is about $+0.035 \mathrm{ppm} / \mathrm{V}$. This tuning can be used for small temperature fluctuations of the oven-controlled resonator package.

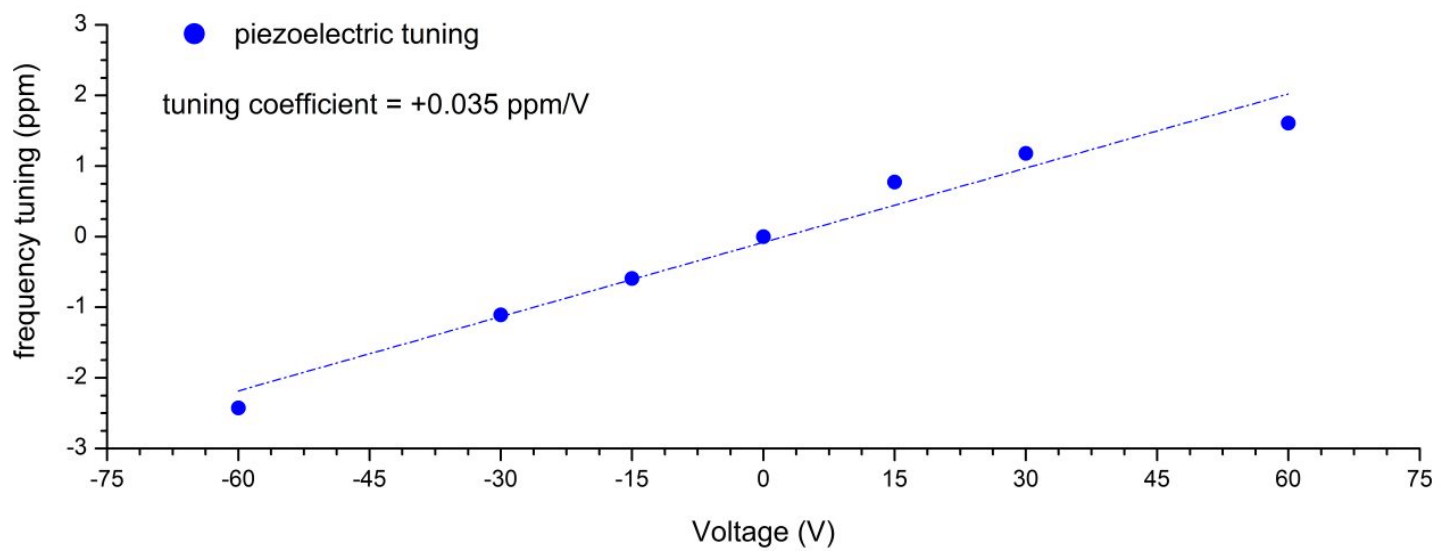

Figure 5. Measured piezoelectric tuning for the AlN-on-silicon in-plane flexural mode IBAR. The low tuning coefficient can be attributed to the relatively small thickness $(0.5 \mu \mathrm{m})$ of the AIN layer as opposed to the thickness of the silicon layer $(20 \mu \mathrm{m})$.

\subsection{Intrinsic Resonator Stability}

Active compensation in addition to passive compensation can be used to achieve highly stable resonators which can be used in low-jitter timing references. However, the ultimate clock performance is determined by the resonator intrinsic drift. In order to measure the intrinsic drift of a resonator, a feedback control loop is implemented to keep the resonator frequency constant. The measurement chamber temperature is maintained within $\pm 100 \mathrm{mK}$. The frequency drift of the resonator is compensated for using electrostatic tuning. As the resonator frequency deviates from the setpoint, the bias is
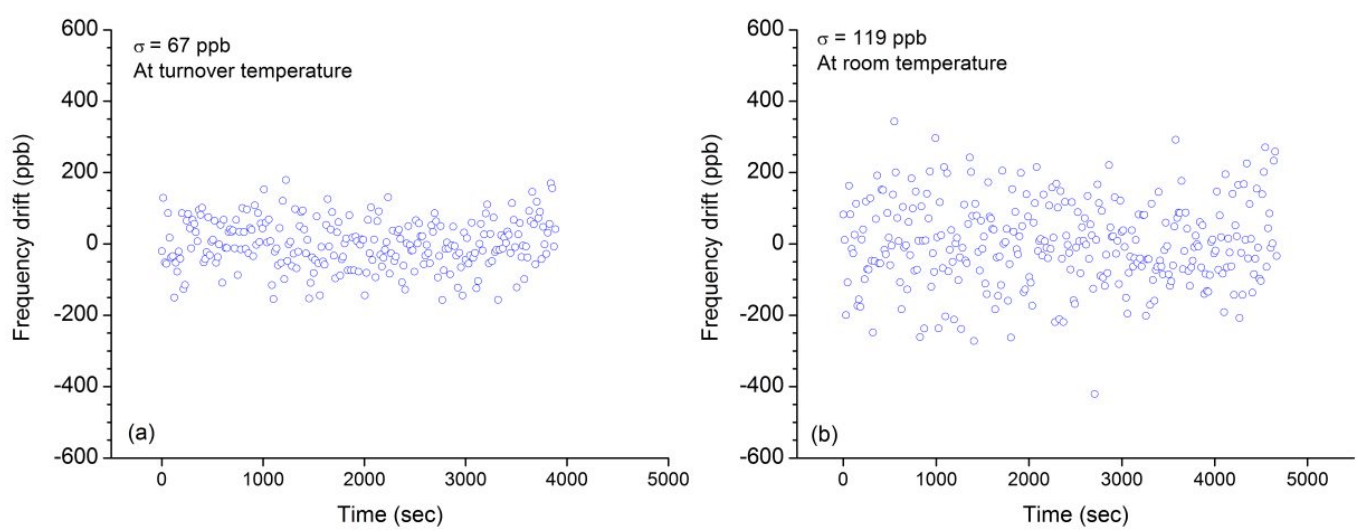

Figure 6. Frequency stability of the resonator with $5.5 \mu \mathrm{m}$ wide oxide trenches, showing a smaller standard deviation when operated near the turnover temperature. 
correspondingly increased or reduced so as to maintain the set frequency. Since, such a compensation scheme can only get rid of correlated drift terms, the measured frequency instability is a metric of the resonator intrinsic drift. Figure 6 plots the measured frequency instability for a compensated device operated at both its turnover temperature and at room temperature. From the results in Fig. 6, it is seen that the frequency stability is better at turnover temperature as opposed to some other temperature (room temperature) away from the turnover point. This result suggests that the drift is due to our inability to maintain a constant measurement system temperature. More work is essential before any specific conclusions can be drawn from these results.

\subsection{Space Applications}

Integrated timekeeping systems with ultra-high accuracy and frequency stability performance are needed in a number of space applications. Such systems address the technical challenges for space clocks, namely sensitivity to on-board thermal environment conditions and long-term and short-term stability problems. The presented micromechanical resonators are both passively and actively compensated to reach unprecedented frequency accuracy and stability with applications in small satellites, such as nano and picosatellites. Picosatellies, i.e. spacecraft that weigh between 1 and 10 $\mathrm{kg}$ and are about $5 \times 5 \times 10 \mathrm{in}^{3}$, are drawing increasing interest as platforms for conducting on-orbit science. This trend is primarily driven by the ability to attach the pico (or nano) satellite on the launch of a larger spacecraft and hence achieve orbit at greatly reduced cost [22]. For such small and low-weight satellites the use of atomic clocks is prohibitive. Therefore, a technology is needed that offers frequency accuracy reaching that of atomic clocks, while also offering a small size, weight, and low power. The presented MEMS resonators are excellent candidates for such applications. Lowpower clocks can be implemented using the TCF-compensated silicon resonators to reach excellent frequency stability, while consuming 5 to 6 orders of magnitude lower power compared to atomic clocks. In addition to picosatellites, precision chip-scale clocks have application in many other space and consumer systems, such as in Global Positioning Systems (GPS) [23]. GPS receivers are vastly used in geodesy and Earth monitoring as well as for the comparison of distant clocks and for the generation of international time scales.

\section{CONCLUSIONS}

In this paper, we demonstrated temperature-stable piezoelectrically actuated and tuned silicon resonators. The first-order TCF of the AlN-on-silicon resonators were compensated using both active and passive compensation techniques. By positioning the compensating oxide-refilled trenches, we demonstrated that the turnover temperature of the resonators can be controlled. Further, by operating the resonator around the turnover temperature, the frequency stability of the resonator is improved. These features make such resonators promising candidates for use in timing applications.

\section{ACKNOWLEDGMENT}

This work was funded by DARPA under award \#N66001-11-C-4170. Devices were fabricated at the Lurie Nanofabrication Facility, a member of the National Nanotechnology Infrastructure Network, which is supported in part by the National Science Foundation.

\section{REFERENCES}

[1] Li, T., Gianchandani, Y.B., "A high-speed batch-mode ultrasonic machining technology for multi-level quartz crystal microstructures," IEEE/ASME International Conference on Micro Electro Mechanical Systems,348-401 (2010).

[2] Rapp, H., Hjort, H.K.,"Cut-independent quartz resonator micromachining by ion track lithography," IEEE International Frequency Control Symposium, 485-488 (1999).

[3] Xie, Y., Li, S., Lin, Y., Ren, Z., Nguyen, C., "1.52-GHz micromechanical extensional wine-glass mode ring resonators," IEEE Trans. Ultrasonics, Ferroelectrics and Frequency Control, 55, 890-907 (2008).

[4] Miri Lavasani, H., Samarao, A. K., Casinovi, G., Ayazi, F. "A 145MHz low phase noise capacitive silicon micromechanical resonator," Tech. Dig. IEEE Int. Electron Device Meeting, pp. 675-678 (2008).

[5] Weinstein, D., Bhave, S.A., "Internal dielectric transduction of a $4.5 \mathrm{GHz}$ silicon bar resonator," IEEE International Electron Devices Meeting, 415-418 (2007). 
[6] Pourkamali, S., Ayazi, F. "Fully single crystal silicon resonator with deep-submicron dry-etched transducer gaps," Proc. IEEE Micro Electro Mechanical Systems Conference, 813-816 (2004).

[7] Harrington, B.P., Abdolvand, R. "Q-Enhancement through minimization of acoustic energy radiation in micromachined lateral-mode resonators," Solid-State Sensors, Actuators and Microsystems Conference, 700 - 703 (2009).

[8] Ziaei-Moayyed, M., Quévy, E.P., Hsieh, J., Howe, R.T., "Efficient internal electrostatic transduction of the 41st radial mode of a ring resonator," IEEE Micro Electro Mechanical Systems Conference, 24 - 28 (2010).

[9] Hung, L.W., Jacobson, Z.A., Ren, Z., Javey, A., Nguyen, C., "Capacitive transducer strengthening via ALD-enabled partial-gap filling," Solid-State Sensors, Actuators and Microsystems Workshop Technical Digest, (2008).

[10] Piazza, G., Stephanou, P.J., Pisano, A.P., "AlN contour-mode vibrating RF MEMS for next generation wireless communications", Proceedings of the 32nd European Solid-State Circuits Conference, 62-65 (2006).

[11] Ruby, R., Barfknecht, A., Han, C.H., Desai, Y., Geefay, F., Gat, M., Verhoeven,T. "High-Q FBAR filters in a wafer level chip scale package," ISSCC, (2002).

[12] Pan, W., Ayazi, F., "Thin-film piezoelectric-on-substrate resonators with Q enhancement and TCF reduction," IEEE International Conference on Micro Electro Mechanical Systems, 104-107 (2010).

[13] Abdolvand, R., Mirilavasani, H., Ho, G.K., Ayazi, F., "Thin-film piezoelectric-on-silicon resonators for highfrequency reference oscillator applications," IEEE Transactions on Ultrasonics Ferroelectrics and Frequency Control 55(12), 2596-2606 (2008).

[14] Ruby, R. "FBAR-from technology development to production", 2nd International Symposium on Acoustic Wave Devices for Future Mobile Communication Systems, 133 (2004).

[15] Samarao, A.K., Ayazi, F., "Temperature compensation of silicon micromechanical resonators via degenerate doping," IEEE International Electron Devices Meeting, 789-792 (2009).

[16] Lin, C.-M., Yen, T.-T., Felmetsger, V.V., Hopcroft, M. A., Kuypers, J. H., Pisano,A.P., "Thermally compensated aluminum nitride Lamb wave resonators for high temperature applications," Applied Physics Letter 97, 083501 (2010).

[17] Melamud, R., et al., "Temperature-compensated high-stability silicon resonators," Applied Physics Letters 90(24), 244107 (2007).

[18] Tabrizian, R., Casinovi, G., Ayazi, F., "Temperature-stable high-Q AlN-on-silicon resonators with embedded array of oxide pillars," Solid-State Sensors, Actuators, and Microsystems Workshop, 100-101 (2010).

[19] Thakar, V., Wu, Z., Peczalski, A., Rais-Zadeh, M., "Piezoelectrically transduced temperature-compensated flexuralmode silicon resonators," IEEE Journal of Microelectromechanical Systems, (2013).

[20] Ho, G.K., Sundaresan, K., Pourkamali, S., Ayazi, F., "Micromechanical IBARs: tunable high-Q resonators for temperature-compensated reference oscillators," IEEE Journal of Microelectromechanical Systems 19(3), 503515(2010).

[21] Ho, G.K., Perng,J.K.C., Ayazi, F. "Micromechanical IBARs: Modeling and Process Compensation," IEEE Journal of Microelectromechanical Systems 19(3), 516-525(2010).

[22] Smith, M. W. et al. "ExoplanetSat: detecting transiting exoplanets using a low-cost CubeSat platform." Space Telescopes and Instrumentation 2010: Optical, Infrared, and Millimeter Wave. Ed. Jacobus M. Oschmann et al. San Diego, California, USA: SPIE, (2010).

[23] Cacciapuoti, L. "Atomic clock ensemble in space," Journal of Physics: Conference Series, 327(2011). 